\title{
Methode zur Berechnung des Mittleren Inneren Potentials und deren Anwendung auf $\mathrm{NaCl}, \mathrm{KBr}$ und $\mathrm{Cu}$
}

\author{
Hans F. Bühner und Siegrried Steeb \\ Institut für Sondermetalle am Max-Planck-Institut für Metallforschung, Stuttgart \\ (Z. Naturforschg. 22 a, 1994-1998 [1967] ; eingegangen am 11. August 1967)
}

\begin{abstract}
From recently published analytic approximations to X-Ray scattering factors which were obtained from improved wave functions, the mean inner potential (MIP) was calculated according to the Bетнe formula. With sodium chloride, potassium bromide and copper the values $7.33,8.32$ and 18.43 Volts, respectively, were obtained. All these values are smaller than the experimental values of $8.24,9.05$ and 19.00 Volts, respectively, which were published recently ${ }^{3}$.

The procedure delivers good results with respect to the comparatively simple method of calculation and it has the advantage of being applicable to all kinds of ions and atoms. Furthermore, a method of calculation is given for the case, that the charge cloud covers a greater region than that of one ion.
\end{abstract}

Das gesamte innere Potential in einem Kristall rührt von den Ladungen aller Kerne und Elektronen her und ist daher periodisch mit der Periodizität des Gitters. Die Fourier-Reihe, in welche dieses Potential entwickelt werden kann, hat ein Glied nullter Ordnung, das von der Ortskoordinate unabhängig ist und, pro Volumeneinheit genommen, das Mittlere Innere Potential (MIP) darstellt ${ }^{1,2}$. Im folgenden sollen die in der vorangehenden Arbeit ${ }^{3}$ experimentell bestimmten Werte des MIP auf theoretischem Wege abgeleitet werden.

\section{Berechnung des MIP einer dreidimensional unendlich ausgedehnten Anordnung weit von- einander entfernt liegender Ionen}

Berechnungen des MIP werden meist mit der auf Bethe $^{4}$ zurückgehenden Beziehung durchgeführt:

$$
\bar{\Phi}=\frac{2}{3} \pi e \sum_{i} N_{i} \int_{0}^{\infty} \varrho_{i}(r) r^{4} \mathrm{~d} r
$$

wobei $\bar{\Phi}^{*}$ das MIP, $e$ die Elementarladung, $N_{i}$ die Anzahl der Atome der $i$-ten Atomsorte pro Volumeneinheit und $r^{2} \cdot \varrho_{i}(r)$ die Anzahl von Elementarladungen ist, die sich auf einer Kugelschale mit den Radien $r$ und $r+\mathrm{d} r$ um ein Atom der Sorte $i$ befinden. Dabei wurde vorausgesetzt, daß die Atome

1 Z. G. Pinsker, Electron Diffraction, Butterworth Scientific Publications, London 1953.

2 B. K. Vainshtein, Structure Analysis by Electron Diffraction, Pergamon Press, New York 1964.

3 S. Steeb u. H. F. Bühner, Z. Naturforschg. 22 a, 1990 [1967].

${ }^{4}$ H. A. Bethe, Ann. Phys. 87, 55 [1928]. bzw. Ionen sphärische Symmetrie und abgeschlossene Schalen haben. Um nun mit dieser Beziehung das MIP berechnen zu können, wird die Ladungsdichte $\varrho(r)$ benötigt, welche in der vorliegenden Arbeit aus der Gleichung für den Atomformfaktor $f(k)$ :

$$
f(k)=\int_{0}^{\infty} \varrho(r) \cdot r \frac{\sin (4 \pi k r)}{(4 \pi k)} \mathrm{d} r,
$$

wobei $k=(\sin \Theta) / \lambda$ ist, durch Fourier-Transformation erhalten wird:

$$
\varrho(r)=(32 \pi / r) \int_{0}^{\infty} k f(k) \sin (4 \pi k r) \mathrm{d} k .
$$

Den Anlaß für diese Art der Berechnung der Ladungsdichte gab die Tatsache, daß neuerdings die Atomformfaktoren ${ }^{5}$ sehr genau bekannt sind, und daß sehr gut angepaßte Interpolationsformeln für dieselben vorliegen. Derartige Interpolationsformeln ${ }^{6}$ gelten für $k=0$ bis $k_{0}=2$ und haben folgende Form:

$$
f(k)=\sum_{w=1}^{w} a_{w} \exp \left(-b_{w} k^{2}\right)+c .
$$

Die Zahl der Summanden ist dabei von Atomsorte zu Atomsorte verschieden. Die Werte der Parameter $a_{w}, b_{w}$ und $c$ wurden für $w=1$ bis 4 der Arbeit ${ }^{6}$

\footnotetext{
* Aus satztechnischen Gründen wird im folgenden der Querstrich über dem $\Phi$ (MIP) weggelassen.

5 Intern. Tables for X-Ray Crystallography, Kynoch Press, Birmingham 1962, Vol. III, p. 258 ff.

${ }^{6}$ D. T. Cromer, A. C. Larson u. J. T. W WBer, Acta Cryst. 17, 1044 [1964].
} 
entnommen. Im Falle von $\mathrm{Cl}$ und $\mathrm{Br}$ sind dort nur die Werte für die neutralen Atome angegeben. Um die Atomformfaktorkurve der entsprechenden Ionen zu approximieren, war es in diesen Fällen nötig, in Gl. (4) jeweils drei neue Summanden, d. h. 6 neue Parameter einzuführen. Dies war auch notwendig im Falle des Kaliumions, weil erst dadurch eine Konvergenz von $f$ für $k>2$ erreicht werden konnte. In Tab. 1 sind diese zusätzlich eingeführten Parameter zusammen mit den Ionenradien nach ${ }^{7}$ und

\begin{tabular}{|c|c|c|c|c|c|}
\hline & $\mathrm{Cu}^{+}$ & $\mathrm{Na}^{+}$ & $\mathrm{Cl}^{-}$ & $\mathbf{K}^{+}$ & $\mathrm{Br}^{-}$ \\
\hline & & & 0,19 & $-2,2$ & 1 \\
\hline & & & 0,4686 & 42,1385 & $\quad 1,6685$ \\
\hline \multirow{4}{*}{1} & & & $-0,15$ & 1,2 & $-0,9$ \\
\hline & & & 3,0427 & 11,9687 & 4,5765 \\
\hline & & & 0,96 & - & 0,9 \\
\hline & & & 12,7727 & - & 27,9484 \\
\hline 2 & 1,075 & 0,98 & 1,81 & 1,33 & 1,96 \\
\hline $\overrightarrow{3}$ & 1,413 & 1,136 & 2,098 & 1,598 & 2,354 \\
\hline
\end{tabular}

Tab. 1. 1. Parameter für die Anwendung in Gl. (4) auf $\mathrm{Cl}^{-}$, $\mathrm{K}^{+}$und $\mathrm{Br}^{-} ; 2$. Ionenradien nach ${ }^{7}$ in $\AA$; 3. vergrößerte Ionenradien nach ${ }^{10}$ in $\AA$ für $\mathrm{Cu}^{+}, \mathrm{Na}^{+}, \mathrm{Cl}^{-}, \mathrm{K}^{+}$und $\mathrm{Br}^{-}$.

den vergrößerten Ionenradien, deren Bedeutung später erläutert werden wird, aufgeführt. An dieser Stelle sei bemerkt, daß mit anderen, sehr genauen und bis $k=6$ vorliegenden Atomformfaktorberechnungen ${ }^{8}$ die Interpolationsformeln erweitert und über ihren vorherigen Gültigkeitsbereich hinaus ausgedehnt werden können. Die nach Einsetzen der $f$-Werte aus Gl. (4) in Gl. (3) sich ergebenden bestimmten Integrale sind in ${ }^{9}$ tabelliert, und man erhält schließlich für die Ladungsdichte folgenden Ausdruck:

$$
\varrho=\varrho_{\mathrm{I}}+\varrho_{\mathrm{II}}
$$

mit $\varrho_{\mathrm{I}}=32 \pi\left\{\sum_{w} \cdot a_{w} \sqrt{\pi^{3} / b_{w}{ }^{3}} \cdot \exp \left[\left(-4 \pi^{2} r^{2}\right) / b_{w}\right]\right\}$

und $\varrho_{\mathrm{II}}=(32 \pi / r) c \int_{0}^{\infty} k \sin (4 \pi r k) \mathrm{d} k$.

Beide Beiträge sollen im folgenden getrennt behandelt werden.

Nach Einsetzen des Ausdruckes für $\varrho_{\mathrm{I}}$ in die Bethesche Gleichung (1) und Integration ergibt sich für den Beitrag von $\varrho_{\mathrm{I}}$ zum MIP einer Ionensorte

$$
\Phi_{Q \mathrm{I}}=\frac{1}{4}(e N / \pi) \sum_{w} a_{w} b_{w}
$$

7 Landolt-Börnstein, 6. Auflage, Atomphysik 1, Teil 4, Kristalle, Springer-Verlag, Berlin-Göttingen-Heidelberg 1955. und für einen aus $j$ Ionensorten zusammengesetzten Ionenkristall entsprechend

$$
\Phi_{Q \mathrm{I}}=\frac{1}{4}(e / \pi) \sum_{j} N_{j} \sum_{w} a_{j w} b_{j w} .
$$

Hierbei ist $N_{j}$ die Anzahl der zur $j$-ten Ionensorte gehörenden Ionen.

Aus Gründen der Stetigkeit muß für große Werte von $k$ der Atomformfaktor exponentiell gegen Null gehen, weshalb Gl. (4) nicht unbegrenzt gültig sein kann. Um eine Konvergenz des Integrals in (7) zu erreichen, kann der exponentielle Abfall näherungsweise für eine Abschätzung des Beitrages von $\varrho_{\mathrm{II}}$ zum MIP durch eine Hyperbel der Form

$$
c(k)=c k_{0} / k \quad \text { für } \quad k>k_{0}
$$

angenähert werden. Diese recht gute Näherung liefert bei Integration bis zu einem Ionenradius $R_{\mathbf{0}}$ folgenden Beitrag zum MIP:

$$
\begin{aligned}
& \Phi_{\varrho \mathrm{II}}=\Phi_{k>k_{0}}+\Phi_{k \leq k_{0}} \\
& \quad=\frac{16}{3} \pi e N c\left\{\frac{\sin \left(4 \pi R_{0} k_{0}\right)}{64 \pi^{3} k_{0}{ }^{2}}-\frac{R_{0} \cos \left(4 \pi R_{0} k_{0}\right)}{16 \pi^{2} k_{0}}\right\} .
\end{aligned}
$$

Nach Einsetzen der Zahlenwerte für die einzelnen Substanzen zeigt sich, daß die Größe $\Phi_{o \text { II }}$ recht klein ist, wobei noch bemerkt werden muß, daß diese Abschätzung eher einen zu großen als zu kleinen Wert ergibt.

\section{Gesamtwert des MIP}

Das MIP einer dreidimensional unendlich ausgedehnten Anordnung von Ionen, die so weit voneinander entfernt liegen, daß sich ihre Elektronenhüllen gegenseitig nicht beeinflussen, errechnet sich zu

$$
\Phi=\Phi_{Q_{\mathrm{I}}}+\Phi_{Q^{\mathrm{II}}}
$$

wobei $\Phi_{\varrho \mathrm{I}}$ der Gl. (8) bzw. (9) und $\Phi_{\varrho \mathrm{II}} \operatorname{der} \mathrm{Gl}$. (10) entnommen werden kann.

3. Berechnung des Mittleren Inneren Potentials einer dreidimensional unendlich ausgedehnten Anordnung von Ionen, welche die durch die Kristallstruktur gegebene tatsächliche Entfernung voneinander haben

Bei den bisherigen Berechnungen wurden die Atomformfaktoren und deren Approximationsfor-

8 H. P. Hanson, F. Herman, J. D. Lea u. S. Skillman, Acta Cryst. 17, 1040 [1964].

9 W. Gröbner u. H. Hofreiter, Integraltafeln, Wien 1961. 
meln verwendet, ohne daß geprüft wurde, ob die durch Fourier-Transformation gewonnene Ladungsdichte in ihrer räumlichen Ausdehnung mit den gegebenen Größenordnungen des entsprechenden Kristallgitters verträglich ist. Bei genauer Betrachtung ist zu bemerken, daß einige Exponentialfunktionen weit über die Größe des Ionenradius hinaus noch eine beträchtliche Ladungsdichte liefern. Würde man diese Ladungsdichten nicht korrigieren, dann würde das bedeuten, daß sich die Elektronen in den äußeren Schalen der Ionen überlappten, was sowohl bei den Metallen nach Abgabe der „freien“ Elektronen an das Elektronengas, als auch bei den hier untersuchten typischen Vertretern der Ionenkristalle nicht möglich ist. Es würden sich damit zu große Werte für das MIP ergeben, da nach der Formel von Bethe [vgl. Gl. (1)] Ladungen in großer Entfernung vom Kern durch die Multiplikation mit der vierten Potenz von $r$ den Potentialwert stark vergrößern. Um diesen Fehler zu vermeiden, soll im folgenden die außerhalb eines durch die Ionendimensionen bestimmten Radius $R_{0}$ gelegene Ladung ausgerechnet und in den Bereich von $0<r<R_{0}$ verteilt werden.

Die gesamte von einem Ion herrührende Ladung beträgt

Um den so korrigierten Wert des MIP zu erhalten, einmal $\varrho+\varrho^{\prime \prime}$ eingesetzt werden, wodurch sich fol-

$$
\Phi_{Q \mathrm{I}}=\frac{2}{3} \pi e \sum N_{i}\left[\int_{0}^{R_{0}} \varrho(r) r^{4} \mathrm{~d} r+\left\{\begin{array}{c}
\frac{3}{5} R_{0}{ }^{2} \int_{R_{0}}^{\infty} \varrho(r) r^{2} \mathrm{~d} r \\
R_{0}^{2} \int_{R_{0}}^{\infty} \varrho(r) r^{2} \mathrm{~d} r
\end{array}\right\}\right] .
$$

Dabei gilt (13 a) für gleichmäßige, (13 b) für schalenförmige Verteilung der Überschußladung und es ist zu erwarten, daß der richtige Wert für $\Phi_{Q \mathrm{I}}$ zwischen den durch (13 a) bzw. (13 b) gelieferten Werten liegen wird. Im Gegensatz zum Potentialbeitrag $\Phi_{Q \mathrm{I}}$ erübrigt sich für $\Phi_{\ell \mathrm{II}}$ eine derartige Korrektur, weil dessen Beitrag zum MIP ohnehin verschwindend klein ist.

Für die Berechnung des Ionenradius $R_{0}$ soll die bei TULL ${ }^{10}$ angegebene Methode verwendet werden, bei der das gesamte Kristallvolumen auf die Ionen aufgeteilt wird. Der dabei erhaltene Radius (vgl. Tab. 1) ist dann natürlich etwas größer als der gewöhnliche Ionenradius.

10 V. F. G. TulL, Proc. Roy. Soc. London A 206, 219 [1951].

$$
\begin{aligned}
Q & =\int_{0}^{\infty} \varrho(r) r^{2} \mathrm{~d} r \\
& =\int_{0}^{R_{0}} \varrho(r) r^{2} \mathrm{~d} r+\int_{R_{0}}^{\infty} \varrho(r) r^{2} \mathrm{~d} r .
\end{aligned}
$$

Aus dieser Beziehung kann die Teilladung

$$
\int_{R_{0}}^{\infty} \varrho(r) r^{2} \mathrm{~d} r
$$

berechnet werden.

Diese Ladung erstreckt sich auf das außerhalb des Ionenradius $R_{0}$ liegende Gebiet, wobei für die geometrische Verteilung nunmehr zwei Extremfälle betrachtet werden sollen: Einmal kann die Ladung gleichmäßig ${ }^{10}$ auf das Gebiet zwischen $0 \leqq r \leqq R_{0}$ und zum anderen auf der Kugelschale mit den Radien $R_{0}-\Delta z$ und $R_{0}+\Delta z$ verteilt sein.

Im ersten Fall gilt dann für die Zusatzladung

$$
\varrho^{\prime}=\int_{R_{0}}^{\infty} \frac{3}{R_{0}{ }^{3}} \varrho(r) r^{2} \mathrm{~d} r
$$

und im zweiten Fall

$$
\varrho^{\prime \prime}=\frac{1}{2 \Delta z R_{0}^{2}} \int_{R_{0}}^{\infty} \varrho(r) r^{2} \mathrm{~d} r .
$$

muß in die Bethesche Beziehung einmal $\varrho+\varrho^{\prime}$ und gende Beziehungen ergeben :

\section{Berechnung des MIP für den Fall des Kupfers}

Für die Berechnung des MIP von Kupfer wird angenommen, daß das Kupfergitter aus einwertigpositiven Kupferionen und gleichmäßig verteilten Elektronen besteht. Dabei soll jedes Atom ein Elektron an das Elektronengas abgegeben haben. Sowohl das Ionengitter als auch das Elektronengas leisten entsprechend ihrer Ladungsdichte Beiträge zum MIP, die im folgenden berechnet werden sollen:

Der vom Ionengitter herrührende Anteil zum MIP setzt sich zusammen aus $\Phi_{\rho \mathrm{I}}$ nach Gl. (8) bzw. (13 a) und (13 b) und $\Phi_{\nu \text { II }}$ nach Gl. (10). Die Werte für den Beitrag des Ionengitters zum MIP ergeben sich nach Gln. (8), (13a) und (13b) zu 
16,52; 14,70 bzw. 15,99 Volt, wobei im ersten Fall angenommen wurde, daß die Ionen so weit voneinander weg sind, daß sie sich nicht gegenseitig stören. Im zweiten Fall wurde gleichmäßige Ladungsverteilung und im dritten Fall die Verteilung der sich überlappenden Ladungsanteile auf Kugelschalen angenommen. Der Beitrag $\Phi_{Q \mathrm{II}}$, herrührend vom konstanten bzw. hyperbelförmigen Beitrag in Gl. (10) beträgt im Fall des Kupfers $\Phi_{Q} \mathrm{II}=0,02$ Volt.

Der Beitrag des Elektronengases kann nach Bethe $^{4}$ berechnet werden. Dabei wird angenommen, daß das Elektronengas gleichmäßig über eine Kugel verteilt ist, deren Volumen gleich dem im Kristall auf jedes Atom entfallenden Volumen ist. Als Beitrag des Elektronengases zum MIP erhält man nach ${ }^{4}$

$$
\Phi_{\mathrm{Eg}}=\frac{3}{10} e / R_{0} .
$$

Mit dem in Tab. 1 angegebenen „vergrößerten“ Ionenradius $R_{0}$ ergibt sich $\Phi_{\mathrm{Eg}}=3,058$ Volt. Als Gesamtwert für das MIP von Kupfer erhält man damit zusammen mit den oben angegebenen Potentialwerten für das Ionengitter :

$$
\Phi=(18,43 \pm 0,65) \text { Volt. }
$$

\section{Berechnung des MIP für das $\mathrm{NaCl}$ und das $\mathrm{KBr}$}

Da beide Substanzen Ionenkristalle bilden, können die Beiträge der jeweiligen Ionen zum MIP einfach addiert werden. Die nach dem beschriebenen Verfahren berechneten Werte sind in Tab. 2 dar-

\begin{tabular}{|c|c|c|c|c|c|}
\hline $\begin{array}{l}\text { Ionen- } \\
\text { sorte }\end{array}$ & $\begin{array}{r}\Phi_{\varrho} \\
(8)\end{array}$ & $\begin{array}{l}\text { in Vol } \\
\text { ach Gl } \\
\text { (13a) }\end{array}$ & $\begin{array}{l}\mathrm{t}, \\
(13 \mathrm{~b})\end{array}$ & $\begin{array}{l}\Phi_{\text {III }} \\
\text { in Volt } \\
\text { nach } \\
\text { Gl. (10) }\end{array}$ & $\begin{array}{l}\text { Endergebnis } \\
\text { (in Volt) }\end{array}$ \\
\hline $\mathrm{Na}^{+}$ & 1,35 & 1,28 & 1,32 & 0,01 & $\Phi_{\mathrm{NaCl}}$ \\
\hline $\mathrm{Cl}^{-}$ & 6,56 & 6,04 & 6,31 & $-0,31$ & $=(7,17 \pm 0,15)$ \\
\hline $\mathrm{K}^{+}$ & 5,84 & 2,29 & 2,60 & 0,02 & $=(8,32 \pm 0,24)$ \\
\hline $\mathrm{Br}^{-}$ & 5,98 & 5,69 & 5,86 & 0,08 & $\Phi_{\mathrm{KBr}}$ \\
\hline
\end{tabular}
gestellt.

Tab. 2. Berechnete Werte für das MIP von $\mathrm{Na}^{+}, \mathrm{Cl}^{-}, \mathrm{K}^{+}$und $\mathrm{Br}^{-}$bzw. $\mathrm{NaCl}$ und $\mathrm{KBr}$.

\section{Diskussion der Berechnungsmethode}

Für den Fall der Ionen liegen die Approximationskurven im Bereich $0,2<k<0,6$ unterhalb der tatsächlichen Atomformfaktorkurven. Jedoch wurde dieser Bereich für Chlor, Kalium und Brom beim Überführen der Näherungskurven für die Atome in die Näherungskurven für die Ionen gleichzeitig korrigiert, so daß die Approximationskurven dieser drei Ionensorten den genauen Verlauf wiedergeben. Bei den Näherungskurven für das Natriumion und das Kupferion sind die Abweichungen sehr klein. Eine Überschlagsrechnung bezüglich deren Einfluß auf den Potentialwert zeigt, daß auf eine Korrektur dieser Näherungskurven verzichtet werden kann.

$\mathrm{Da}$ in den Wert des MIP besonders die steil abfallenden, also durch große Werte von $b$ gekennzeichneten Exponentialfunktionen eingehen, muß die Approximationskurve vor allem für kleine $k$ den tatsächlichen Verlauf wiedergeben, was bei allen durchgeführten Rechnungen der Fall ist.

Für große $k$, bei denen die Beiträge der Exponentialfunktionen mehr und mehr verschwinden und die Konstante $c$ stärker ins Gewicht fällt, wird diese "Konstante“ durch eine Hyperbel ersetzt. Abgesehen vom Fall des Chlorions ist diese Annäherung gut. Da die gewählte Hyperbel meist etwas schneller als die Atomformfaktorkurve abfällt, ist der ohnehin schon verschwindend kleine Beitrag, der sich nach Gl. (10) berechnet, in Wirklichkeit noch kleiner und kann damit vernachlässigt werden.

Eine Ausnahme bildet der für das Chlorion berechnete Wert des MIP. Beim Chlorion ist die Konstante $c$ um eine Größenordnung größer als bei den anderen Ionen und außerdem negativ ${ }^{6}$. Bedingt durch die Größe von $c$ fällt die Approximationshyperbel schneller ab als die anzunähernde Atomformfaktorkurve. Eine Abschätzung ergibt, daß dadurch der in Tab. 2 für das MIP des Chlorions angegebene Wert sich um 0 bis 0,3 Volt zu klein ergeben hatte. Danach wäre als endgültiger Wert für das MIP des $\mathrm{NaCl}$ in Tab. $2 \Phi_{\text {korr }}=(7,33 \pm 0,3)$ Volt einzusetzen.

Die vorliegende Art der Berechnung des MIP ist ohne Schwierigkeiten für alle Atome und Ionen anwendbar, für die Approximationskurven vorliegen ${ }^{\mathbf{6}}$, d. h. also für alle Atome und die wichtigsten Ionen der Ordnungszahlen 2-92. Für niedrige Ordnungszahlen kann außerdem auf Grund der kleinen Korrekturen ohne weiteres die vereinfachte Form der Gl. (8) verwendet werden. Für große Ordnungszahlen liefern die angegebenen Ladungsdichtekorrekturen die Möglichkeit, einen verläßlichen Wert zu finden.

Bezüglich der Neuverteilung der über den Ionenradius $R_{0}$ hinausragenden Ladungsanteile ist zu bemerken, daß nach den Ergebnissen zu urteilen bei 
den hier vorliegenden Ionen-Kristallen mit fast rein elektrostatischer Bindung die Änderung der Ladungsverteilung beim Einbau der freien Ionen in das Kristallgitter durch die angewandten Korrekturen gut erfaßt wird. Die Vernachlässigung der in Wirklichkeit sicher vorhandenen Abweichungen der Ionenbegrenzung von der Kugelsymmetrie bewirkt eine geringfügige Verkleinerung der berechneten MIP-Werte. Diese Tatsache spiegelt sich auch beim Vergleich von berechneten und experimentell bestimmten Werten in Tab. 3 wider.

\begin{tabular}{lrccccc}
\hline & $\begin{array}{c}\text { Exp. } \\
\text { nach }\end{array}$ & $\begin{array}{c}\text { Berechnet } \\
\text { nach vor- } \\
\text { liegender } \\
\text { Arbeit }\end{array}$ & $\begin{array}{c}\text { Be- } \\
\text { rechnet } \\
\text { nach10 }\end{array}$ & $\begin{array}{c}\text { Be- } \\
\text { rechnet } \\
\text { nach11 }\end{array}$ & \multicolumn{2}{c}{$\begin{array}{c}\text { Berechnet } \\
\text { nach } 12\end{array}$} \\
TFD & TF \\
$\mathrm{Cu}$ & 19,00 & $18,43 \pm 0,65$ & 13,7 & 20,2 & 20,8 & 23,4 \\
$\mathrm{NaCl}$ & 8,24 & $7,33 \pm 0,3$ & 8,7 & - & - & - \\
$\mathrm{KBr}$ & 9,05 & $8,32 \pm 0,24$ & - & - & - & - \\
\hline
\end{tabular}

Tab. 3. Experimentelle und berechnete Werte des MIP von $\mathrm{NaCl}, \mathrm{KBr}$ und $\mathrm{Cu}$ in Volt.

In Tab. 3 sind außer den in ${ }^{3}$ gemessenen und im Rahmen dieser Arbeit berechneten Ergebnissen noch einige Werte anderer Autoren ${ }^{11}$ angegeben. Eine neuere Rechnung von Anishchenko ${ }^{12}$ liefert MIP. Werte für 49 Metalle, die mit dem statistischen Mo-

11 Y. Sıота, Sci. Rep. Tôhoku Univ. 42, 4, 173 [1958]. dell für das komprimierte Atom nach ThomasFermi-Dirac (TFD) bzw. Thomas-Fermi (TF) berechnet wurden. Der nach der TFD-Methode mit Austauschwechselwirkung berechnete Wert für das Kupfer stimmt dabei am ehesten mit dem hier ermittelten überein.

\section{Zusammenfassung}

Aus den in neuerer Zeit bekannt gewordenen Atomformfaktorkurven, die auf Grund der Kenntnis verbesserter Wellenfunktionen errechnet wurden, wird das Mittlere Innere Potential nach der Betheschen Beziehung berechnet. Im einzelnen ergaben sich für Natriumchlorid, Kaliumbromid und Kupfer die Werte 7,33, 8,32 bzw, 18,43 Volt. Diese Werte liegen durchweg etwas unter den in einer früheren Mitteilung ${ }^{3}$ angegebenen, experimentell bestimmten Werten von 8,24, 9,05 bzw. 19,00 Volt. Das angegebene Verfahren liefert in Anbetracht der einfachen Berechnungsmethode bemerkenswert gute Ergebnisse, wobei es noch den Vorteil hat, daß es ohne wesentliche Modifikationen für alle Ionen- und Atomsorten anwendbar ist. Für den Fall, daß die Ausdehnung der Ladungswolken größer als die der Ionen ist, wird eine Korrekturmöglichkeit angegeben.

12 R. J. Anishchenko, Phys. Status Solidi 18, 923 [1966]. 